\title{
COMMUTING PAIRS OF PATTERNS AND SYMMETRIC REALIZATIONS*
}

\author{
CHARLES R. JOHNSON ${ }^{\dagger}$ AND OLIVIA WALCH!
}

\begin{abstract}
The patterns that commute with tridiagonal and with other tree patterns are studied. New "ratio" equations are given for the entries of a matrix that commutes with an irreducible tridiagonal one, and these equations imply that a pattern commuting with an irreducible tridiagonal one must be combinatorially symmetric. For an irreducible tridiagonal pattern and another pattern that commutes with it, it is shown that there is always a complex symmetric example of commutativity. However, there need not be a real symmetric commuting instance. An 8-by-8 example is given that settles a natural and long-standing question (whether real, commuting, combinatorially symmetric patterns may be realized by real symmetric matrices). Finally, similar results are given for other patterns, in place of irreducible tridiagonal, under additional hypotheses.
\end{abstract}

Key words. Commuting matrices, pattern, zero-nonzero pattern, symmetric realization, ratio equations, tree, tridiagonal pattern

AMS subject classifications. 15A27, 05C50.

1. Introduction. A zero/nonzero pattern (hereafter pattern) is a rectangular array consisting of *'s and 0 's, in which a * is allowed to be any nonzero number over a given field. Thus, we associate with a pattern $\mathcal{P}$ the set of all matrices with zeros and nonzeros in precisely the positions indicated by $\mathcal{P}$; we also use $\mathcal{P}$ to denote this set. We are interested here in $n$-by- $n$ patterns, and we say that two patterns $\mathcal{P}$ and $\mathcal{Q}$ commute if there exist matrices $P \in \mathcal{P}$ and $Q \in \mathcal{Q}$ such that $P$ and $Q$ commute: $P Q=Q P$. Of course, many pairs of patterns do commute, but it is easy to construct two patterns that do not allow commutativity; see [DJ] for past work on this topic.

Much conventional matrix terminology is easily transferred to patterns, and we often manipulate patterns as combinatorial objects much like matrices. We are particularly interested in pairs of patterns, one of which is the full, irreducible, tridiagonal pattern $\mathcal{T}$, i.e. the only zero entries of $\mathcal{T}$ are those outside the tridiagonal part. (Irreducibility is assumed in all references to the tridiagonal pattern that follow.) The commutant $C(\mathcal{P})$ of a pattern $\mathcal{P}$ is the collection of all patterns that commute with $\mathcal{P}$; a combinatorially symmetric pattern is one in which the nonzero entries are symmetrically arranged. We will, in particular, be interested in those combinatorially

${ }^{*}$ Received by the editors on December 31, 2011. Accepted for publication on August 24, 2012 Handling Editor: Natalia Bebiano.

${ }^{\dagger}$ Department of Mathematics, College of William and Mary, Williamsburg, VA, 23187 USA (crjohnso@math.wm.edu and ojwalch@email.wm.edu). 
symmetric patterns the undirected graph of whose *'s is a tree. However, in neither case do we necessarily restrict the realizing matrices in the corresponding set to be symmetric.

A natural question arises about pairs of combinatorially symmetric patterns. If they commute over the reals, can the commutativity be realized by a pair of real symmetric matrices? Such an instance is referred to hereafter as real symmetric commutativity of patterns. Other work $[\mathrm{S}]$ has shown that all combinatorially symmetric pairs of real commuting patterns of size 3 -by-3 and less also real symmetric commute. This is also so for the commutant of $\mathcal{T}$ for $n=4$. It was reasonable to conjecture that this be so in general, and this question has been open for some time.

In Section 2 we derive some interesting structure in the commutant of the tridiagonal pattern $\mathcal{T}$, and give important numerical formulae relating the entries of commuting matrices, one of which is tridiagonal. In particular, every pattern in $C(\mathcal{T})$ is combinatorially symmetric. Similar results are derived in Section 3 for tree patterns under slight additional assumptions.

For the tridiagonal pattern, we show in Section 5 that for every element $\mathcal{P}$ of the commutant, there are complex symmetric matrices $T \in \mathcal{T}$, and $P \in \mathcal{P}$ that realize the commutativity (in keeping with our terminology, that allow complex symmetric commutativity of the two patterns). However, this is not generally true over the reals. An 8-by-8 pattern $\mathcal{P}$ is given for which $\mathcal{T}$ and $\mathcal{P}$ commute over the reals, but, provably, the commutativity cannot be realized by real symmetric matrices. This leaves the question of whether the smallest such examples occur for $n=5,6,7$, or 8 , and for which elements of the commutant there are real symmetric commuting pairs.

2. The Ratio Equations for Tridiagonal Matrices. Complex symmetric commutativity in the tridiagonal commutant is guaranteed by the results below. As it is used in the following, symmetrizable by a diagonal matrix describes any matrix $A$ that can be written in the form $A=D S$, in which $D$ is an invertible diagonal matrix and $S$ is symmetric. It is equivalent to write $A=D S D D^{-1}=(D S D) D^{-1}=R D^{-1}$, with $R$ symmetric; thus, the left or right placement of $D$ does not matter.

Lemma 2.1. Let $A \in M_{n}(\mathbb{F})$ have pattern $\mathcal{P}$ and $B \in M_{n}(\mathbb{F})$ have pattern $\mathcal{Q}$, with $A$ and $B$ commuting. If $A$ is nonderogatory and symmetrizable by $D \in M_{n}(\mathbb{F})$, then

(a) $B$ is symmetrizable by $D$;

(b) $\mathcal{P}$ and $\mathcal{Q}$ are combinatorially symmetric patterns; and

(c) $\mathcal{P}$ and $\mathcal{Q}$ symmetrically commute, possibly over an extension field of $\mathbb{F}$.

Proof. Because $A$ is symmetrizable by $D$, we know that $A=D S$. We know furthermore (because $A$ is nonderogatory) that if $B$ commutes with $A$, then $B$ can 
be written as a polynomial in $A$, say $B=p(A)[\mathrm{HJ}]$.

So:

$$
\begin{aligned}
B & =p(A) \\
& =\alpha_{n-1} A^{n-1}+\alpha_{n-2} A^{n-2}+\cdots+\alpha_{0} I \\
& =\alpha_{n-1}(D S)^{n-1}+\alpha_{n-2}(D S)^{n-2}+\cdots+\alpha_{0}\left(D D^{-1}\right) \\
& =D\left(\alpha_{n-1}(S D \cdots D S)+\alpha_{n-2}(S D \cdots D S)+\cdots+\alpha_{0}\left(D^{-1}\right)\right) \\
& =D R
\end{aligned}
$$

in which $R$ is symmetric. So $B$ is symmetrizable by $\mathrm{D}$.

Statement (b) can be verified by observing that any matrix that is symmetrizable by an invertible diagonal matrix must be combinatorially symmetric. For $A$, this is given; for $B$, this is shown above.

For (c), the assumption that $A B=B A$ implies $D S D R=D R D S$.

Multiply on the left by $D^{-1 / 2}$ and on the right by $D^{1 / 2}$ with $\left(D^{1 / 2}\right)^{2}=D$ and $D^{-1 / 2}=\left(D^{1 / 2}\right)^{-1}$. Those matrices necessarily exist, though perhaps over an extension field of $\mathbb{F}$. Then

$$
\begin{aligned}
D^{1 / 2} S D R D^{1 / 2} & =D^{1 / 2} R D S D^{1 / 2} \\
\left(D^{1 / 2} S D^{1 / 2}\right)\left(D^{1 / 2} R D^{1 / 2}\right) & =\left(D^{1 / 2} R D^{1 / 2}\right)\left(D^{1 / 2} S D^{1 / 2}\right) .
\end{aligned}
$$

Let $F=D^{1 / 2} S D^{1 / 2}$ and $G=D^{1 / 2} R D^{1 / 2}$. Then $F G=G F$, and $F$ has pattern $\mathcal{P}$ while $G$ has pattern $\mathcal{Q}$. So $\mathcal{P}$ and $\mathcal{Q}$ symmetrically commute.

TheOREM 2.2. Any pattern that commutes with an irreducible tridiagonal pattern must be combinatorially symmetric.

Proof. Every matrix of the irreducible tridiagonal pattern is necessarily nonderogatory. By Lemma 2.1, this implies that any matrix that commutes with a tridiagonal matrix must be combinatorially symmetric. Then any pattern that commutes with the tridiagonal pattern must be combinatorially symmetric as well. $\mathbf{D}$

Theorem 2.3. For any matrix $A=\left[a_{i j}\right]$ with tridiagonal pattern $\mathcal{T}$ and any commuting matrix $B=\left[b_{i j}\right]$,

$$
\frac{b_{i j}}{b_{j i}}=\prod_{k=i}^{j-1} \frac{a_{k k+1}}{a_{k+1 k}} .
$$


Thus, the ratio between any two symmetrically placed off-diagonal entries in a commuting matrix for a given tridiagonal matrix can be expressed as a product of like ratios from the original tridiagonal matrix. This is a special case of a more general result that will be proven in Section 4.

3. Other Trees. That every matrix of the irreducible tridiagonal pattern is necessarily nonderogatory is a property not shared by other trees. In the example below, a not nonderogatory matrix whose graph has the "star" pattern is shown to commute with a combinatorially asymmetric matrix.

EXAMPLE 3.1.

$\left[\begin{array}{llll}1 & 1 & 1 & 1 \\ 2 & 2 & 0 & 0 \\ 2 & 0 & 2 & 0 \\ 2 & 0 & 0 & 2\end{array}\right]\left[\begin{array}{cccc}1 & 1 & 1 & 1 \\ 2 & 3 & 1 & -2 \\ 2 & 0 & 3 & -1 \\ 2 & -1 & -2 & 5\end{array}\right]-\left[\begin{array}{cccc}1 & 1 & 1 & 1 \\ 2 & 3 & 1 & -2 \\ 2 & 0 & 3 & -1 \\ 2 & -1 & -2 & 5\end{array}\right]\left[\begin{array}{llll}1 & 1 & 1 & 1 \\ 2 & 2 & 0 & 0 \\ 2 & 0 & 2 & 0 \\ 2 & 0 & 0 & 2\end{array}\right]=0$

In fact, the tree corresponding to the tridiagonal matrix is the only graph with path cover number $P=1$, which by the result in $[\mathrm{JL}]$ makes the tridiagonal pattern the only pattern whose graph is a tree for which all matrices of the pattern are necessarily nonderogatory. Thus, while the results described here for the tridiagonal pattern hold for nonderogatory matrices of other patterns whose graphs are trees, they are not valid for all matrices of such patterns.

4. Simultaneous Symmetrizability. A generalization of the ratio equations holds true for matrices that are not trees but are simultaneously symmetrizable.

Theorem 1. Let $A=\left[a_{i j}\right] \in M_{n}$ and $B=\left[b_{i j}\right] \in M_{n}$ be two combinatorially symmetric matrices that are simultaneously symmetrizable. Then

$$
\frac{a_{k_{1} k_{2}} a_{k_{2} k_{3}} \ldots a_{k_{m-1} k_{m}}}{a_{k_{2} k_{1}} a_{k_{3} k_{2}} \ldots a_{k_{m} k_{m-1}}}=\frac{b_{k_{1} k_{2}^{\prime}} b_{k_{2}^{\prime} k_{3}^{\prime}} \ldots b_{k_{l}^{\prime} k_{m}}}{b_{k_{2}^{\prime} k_{1}} b_{k_{3}^{\prime} k_{2}^{\prime}} \ldots b_{k_{m} k_{l}^{\prime}}}
$$

for all $k_{i}$ and $k_{i}^{\prime}$ (where the entries of $A$ and $B$ that appear are assumed to be nonzero).

Proof. This result comes easily from simultaneous symmetrizability. If $A$ and $B$ are simultaneously symmetrizable, then $A=D S$ and $B=D R$ where $S$ and $R$ are symmetric. Assume $D=\operatorname{diag}\left(d_{k k}\right)$. Then for all nonzero entries $a_{i j}$ and $b_{p q}$ in $A$ and $B, \frac{a_{i j}}{a_{j i}}=\frac{d_{i i}}{d_{j j}}$ and $\frac{b_{p q}}{b_{q p}}=\frac{d_{p p}}{d_{q q}}$.

For any walk $k_{1}, k_{2}, \ldots, k_{m}$ in the indices of $A$,

$$
\frac{a_{k_{1} k_{2}} a_{k_{2} k_{3}} \ldots a_{k_{m-1} k_{m}}}{a_{k_{2} k_{1}} a_{k_{3} k_{2}} \ldots a_{k_{m} k_{m-1}}}=\frac{d_{k_{1} k_{1}}}{d_{k_{2} k_{2}}} \frac{d_{k_{2} k_{2}}}{d_{k_{3} k_{3}}} \ldots \frac{d_{k_{m-1} k_{m-1}}}{d_{k_{m} k_{m}}}=\frac{d_{k_{1} k_{1}}}{d_{k_{m} k_{m}}}
$$


The same result holds for any walk $k_{1}, k_{2}^{\prime}, k_{3}^{\prime}, \ldots, k_{l}^{\prime}, k_{m}$ in $B$; thus the ratios of the two walks are equal.

5. Symmetry of Realizations in the Tridiagonal Commutant. Recall the question of whether or not for every combinatorially symmetric pair of real commuting patterns there exists a real symmetric pair of real commuting patterns; the following section shows that this is not the case.

THEOREM 2. If pattern $\mathcal{P}$ commutes with an irreducible tridiagonal pattern $\mathcal{T}$, then $\mathcal{P}$ complex symmetric commutes with $\mathcal{T}$.

Proof. If $\mathcal{P}$ commutes with $\mathcal{T}$, then there exist $B$ with pattern $\mathcal{P}$ and $A$, irreducible tridiagonal, such that $A$ and $B$ commute. $A$ is necessarily nonderogatory; thus by Lemma 2.1, we know that a complex symmetric commuting pair of matrices must exist with patterns $\mathcal{P}$ and $\mathcal{T}$.

EXAMPLE 5.1. If pattern $\mathcal{P}$ real commutes with $\mathcal{T}$, then $\mathcal{P}$ need not real symmetric commute with $\mathcal{T}$.

Consider the pattern

$$
\mathcal{B}=\left[\begin{array}{llllllll}
0 & * & 0 & 0 & 0 & 0 & 0 & * \\
* & 0 & * & 0 & 0 & 0 & * & * \\
0 & * & 0 & * & 0 & * & 0 & * \\
0 & 0 & * & * & * & 0 & * & * \\
0 & 0 & 0 & * & * & * & 0 & 0 \\
0 & 0 & * & 0 & * & 0 & * & * \\
0 & * & 0 & * & 0 & * & 0 & 0 \\
* & * & * & * & 0 & * & 0 & *
\end{array}\right] .
$$

Let $B=\left[b_{i j}\right]$ be a matrix of pattern $\mathcal{B}$ and assume it commutes with $A=\left[a_{i j}\right]$, where $A$ is tridiagonal. Define $C$ to be $C=A B-B A$, where the entries of $C$ are expressions (equal to zero) in terms of the entries of $A$ and $B$.

The $(1,2)$ entry of $C$ is

$$
a_{11} b_{12}-b_{12} a_{22}=0
$$

Because $b_{12}$ must be nonzero (for $B$ to be have the pattern $\mathcal{B}$ ), the equality $a_{11}=a_{22}$ 
must hold for any tridiagonal $A$ that permits commutativity with a matrix of pattern $\mathcal{B}$.

Similarly, the $(2,3)$ and $(3,6)$ entries of $C$ contain the equations

$$
\begin{aligned}
& a_{22} b_{23}-b_{23} a_{33}=0 \\
& a_{33} b_{36}-b_{36} a_{66}=0,
\end{aligned}
$$

which require that $a_{22}=a_{33}$ and $a_{33}=a_{66}$. So $a_{11}=a_{22}=a_{33}=a_{66}$ must be true in the tridiagonal or commutativity with $B$ cannot occur.

The $(1,8)$ entry of $C$ is $b_{18}\left(a_{11}-a_{88}\right)+a_{12} b_{28}=0$, and thus

$$
b_{18}\left(a_{11}-a_{88}\right)=-a_{12} b_{28} \text {. }
$$

Since $a_{12}$ and $b_{2,8}$ are nonzero, $a_{22}-a_{77}$.

Likewise, the $(2,7)$ entry of $C$ is $\left(a_{22}-a_{77}\right) b_{27}-b_{28} a_{87}=0$, and thus

$$
\left(a_{22}-a_{77}\right) b_{27}=b_{28} a_{87} .
$$

Since $a_{8,7}$ and $b_{2,8}$ are nonzero, $a_{22}-a_{77}$ is nonzero.

We can therefore divide each side of (5.3) by those of (5.2) to produce

$$
\frac{\left(a_{22}-a_{77}\right) b_{27}}{\left(a_{11}-a_{88}\right) b_{18}}=\frac{-a_{87}}{a_{12}}
$$

Equation $\left(5.4\right.$ can be reduced further by noting that the $(1,7)$ entry of $C$ is $a_{12} b_{27}-$ $b_{18} a_{87}=0$, or

$$
\frac{b_{27}}{b_{18}}=\frac{a_{87}}{a_{12}} .
$$

These terms thus cancel, yielding another equality that must hold in any tridiagonal that commutes with $B$ :

$$
\left(a_{22}-a_{77}\right)=-\left(a_{11}-a_{88}\right)
$$

Similar manipulation of entries $(6,7)$ and $(6,8)$ yields the equations

$$
\begin{aligned}
\left(a_{66}-a_{77}\right) b_{67} & =b_{68} a_{87} \\
b_{67} a_{78} & =\left(a_{66}-a_{88}\right) b_{68}
\end{aligned}
$$

Again dividing one equation into the other produces

$$
\frac{a_{78}}{\left(a_{66}-a_{77}\right)}=\frac{\left(a_{66}-a_{88}\right)}{a_{87}}
$$


Because $a_{11}=a_{22}=a_{33}=a_{66}$ and $\left(a_{22}-a_{77}\right)=\left(a_{11}-a_{77}\right)=-\left(a_{11}-a_{88}\right)$, we can make appropriate substitutions and rewrite the equation as

$$
-\left(a_{11}-a_{77}\right)^{2}=a_{78} a_{87} .
$$

If $A$ is symmetric, then $a_{78}=a_{87}$ and $A$ must contain complex entries. Therefore, no real symmetric matrix that commutes with a real symmetric tridiagonal can have pattern $\mathcal{B}$.

It remains to show that there is a real matrix of pattern $\mathcal{B}$ and a real, irreducible tridiagonal matrix that commutes with it. In fact, both may be chosen rational.

$$
\begin{aligned}
& A=\left[\begin{array}{cccccccc}
1 & 1 & 0 & 0 & 0 & 0 & 0 & 0 \\
\frac{3}{2} & 1 & 1 & 0 & 0 & 0 & 0 & 0 \\
0 & \frac{1}{2} & 1 & 1 & 0 & 0 & 0 & 0 \\
0 & 0 & -\frac{3}{5} & \frac{8}{5} & 1 & 0 & 0 & 0 \\
0 & 0 & 0 & \frac{73}{30} & \frac{3}{4} & 1 & 0 & 0 \\
0 & 0 & 0 & 0 & -\frac{19}{24} & 1 & 1 & 0 \\
0 & 0 & 0 & 0 & 0 & -\frac{1}{2} & 4 & 1 \\
0 & 0 & 0 & 0 & 0 & 0 & -9 & -2
\end{array}\right] \\
& B=\left[\begin{array}{cccccccc}
0 & \frac{369}{104} & 0 & 0 & 0 & 0 & 0 & \frac{2}{13} \\
\frac{1107}{208} & 0 & \frac{369}{104} & 0 & 0 & 0 & -\frac{18}{13} & -\frac{6}{13} \\
0 & \frac{369}{208} & 0 & \frac{369}{104} & 0 & \frac{9}{13} & 0 & -\frac{3}{13} \\
0 & 0 & -\frac{1107}{520} & \frac{1107}{520} & 3 & 0 & \frac{45}{13} & \frac{12}{13} \\
0 & 0 & 0 & \frac{73}{10} & -\frac{219}{520} & \frac{219}{130} & 0 & 0 \\
0 & 0 & \frac{4161}{5200} & 0 & -\frac{1387}{1040} & 0 & -\frac{438}{65} & -\frac{146}{65} \\
0 & \frac{4161}{10400} & 0 & \frac{1387}{416} & 0 & \frac{219}{65} & 0 & 0 \\
\frac{12483}{20800} & -\frac{12483}{10400} & -\frac{12483}{10400} & -\frac{4161}{520} & 0 & -\frac{657}{65} & 0 & -\frac{73}{65}
\end{array}\right]
\end{aligned}
$$

We do not know if this is the smallest order of such example, but it does not appear that a smaller example can be constructed in the same way. 
Electronic Journal of Linear Algebra ISSN 1081-3810

A publication of the International Linear Algebra Society

Volume 25, pp. 84-91, August 2012

\section{REFERENCES}

[DJ] da Graca Marques, M. and Johnson, C. R. "Patterns of commutativity: the commutant of the full pattern." Electron. J. Linear Algebra 14:43-50 (2005).

[HJ] Horn, R. and Johnson, C. R. Matrix Analysis. Cambridge University Press (1990).

[JL] Johnson, C. R. and Leal-Duarte, A. "The maximum multiplicity of an eigenvalue in a matrix whose graph is a tree." Linear and Multilinear Algebra 46:139-144 (1999).

[S] Private communication of joint work of Michael Schilling and C.R. Johnson. 